\title{
CLASSIFICATION OF MAGNETOATMOSPHERIC MODES IN SUNSPOT UMBRAE
}

\author{
S.S.HASAN \\ Indian Institute of Astrophysics \\ Bangalore 560034,India
}

\author{
Y.SOBOUTI \\ Physics Department \\ Shiraz,Iran
}

\begin{abstract}
We examine the wave modes in a sunspot umbra. Assuming a stratification, based on a model atmosphere in a sunspot, the normal mode spectrum is determined. The modes are classified using a scheme based on a Helmholtz decomposition of the displacements into l(longitudinal) and $t$ (transverse) components. In certain cases these can be related to the usual fast and slow MHD waves. We compute the theoretical eigenfrequencies and note the existence of umbral oscillations with periods in the range 2-3 min, which are interpreted as slow and mixed modes. The frequencies of the Alfven waves are also calculated. It is suggested that these modes might also have been observed.
\end{abstract}

\section{Introduction}

Oscillations in the umbrae of sunpots have been widely reported (e.g. Beckers and Schulz,1972; Balthasar and Wiehr,1984; Lites and Thomas,1985; Lites,1986; Abdelatif et al.,1986;Thomas et al. ,1987 and Gurman,1987). The periods of these oscillations typically lie in the range 2-3 min, although larger periods of some 300-400 s (Bhatnagar et al.,1972; Soltau et al.,1976 and Balthasar et al.,1987) or smaller ones around $100 \mathrm{~s}$ (Schröter and Soltau,1976) cannot be ruled out.

A number of theoretical investigations have been carried out to examine the nature of wave propagation in sunpot umbrae (e.g. Uchida and Sakurai,1975; Scheuer and Thomas,1981; Thomas and Scheuer,1982 and Leroy and Schwartz,1982). The interpretation of the observed modes is still not established. Scheuer and Thomas (1981) suggested that umbral oscillations are essentially fast waves trapped in a photopheric cavity. On the other hand, Zhugzhda et al. (1983) and Gurman and Leibacher (1984) have argued in favour of a slow mode, trapped in a chromospheric cavity above the temperature minimum. The aim of the present analysis is to look carefully into this question. Our plan is first to calculate the normal modes of a model sunspot atmosphere with a uniform vertical magnetic field. A classification of these modes is then attempted, based upon a technique developed earlier by us (Hasan and Sobouti,1987; henceforth HS). We also discuss the connection between the modes, classified according to our scheme, and the conventional MHD modes of an unstratified medium.

\section{Equations and Method}

\subsection{EQUILIBRIUM UMBRAL MODEL}

In order to compute the wave modes in an umbra, we require an equilibrium model which mimics a real atmosphere in a sunspot. We selected the core umbral model M of Maltby et al. (1985) (kindly provided to us by T. Abdelatif). Beneath the photosphere, the atmosphere is matched to a convection zone model. Figure 1 shows the temperature variation with $\mathrm{z}$ (depth) in a sunspot. The convention used is that $\mathrm{z}$ increases into the Sun and $\mathrm{z}=0$ corresponds to optical depth unity. 


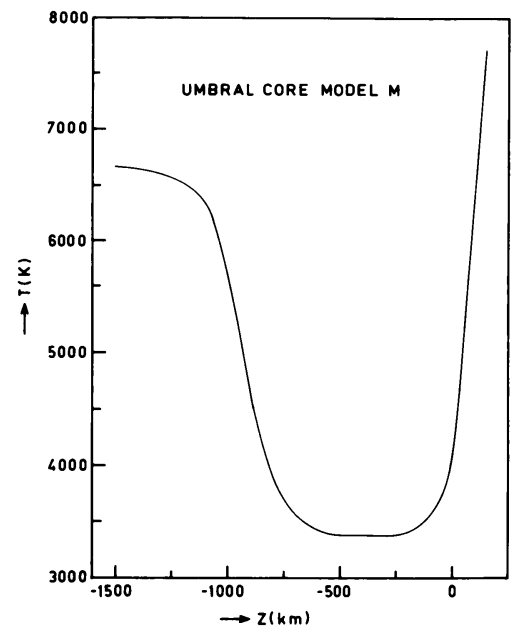

Figure 1. Temperature as a function of $z$ in a sunspot umbra (based on the core umbral model $\mathrm{M}$ of Maltby et al.).

\subsection{LINEARIZED EQUATIONS}

We assume that a uniform vertical magnetic field is embedded in the model atmosphere. The normal modes can be determined by solving the wave equation

$$
\rho \frac{\partial^{2} \xi}{\partial t^{2}}=-\nabla \delta p+\mathbf{g} \delta \rho+\frac{1}{4 \pi}(\nabla \times \delta \mathbf{B}) \times \mathbf{B}
$$

where $\delta \rho, \delta p, \delta \mathbf{B}$ denote perturbations in density, pressure and magnetic field respectively and $\xi$ is a small displacement of a fluid element. Equation (1) can be recast into an energy integral, which lends itself to a variational treatment (for details see HS). Assuming a time dependence of the form $e^{i \omega t}$, the normal frequencies can be calculated using a Rayleigh Ritz variational method.

\subsection{MODE CLASSIFICATION}

In order to classify the modes, we decompose $\zeta$, where $\zeta$ is a linear displacement, using a modified form of Helmholtz' theorem (Sobouti 1981), as follows

$$
\zeta=\zeta_{l}+\zeta_{t}+\zeta_{a}
$$

where the various components can be expressed in terms of scalar functions $\chi$ as

$$
\zeta_{l}=-\nabla \chi_{l} \quad, \quad \zeta_{t}=\frac{1}{\rho} \nabla \times \nabla \times\left(\widehat{z} \chi_{t}\right) \quad \text { and } \quad \zeta_{a}=\nabla \times \nabla \times \nabla \times\left(\widehat{z} \chi_{a}\right)
$$

The irrotational vector $\zeta_{l}$ is associated with longitudinal motions, whereas the remaining two are essentially solenoidal transverse displacements. It turns out that $\zeta_{a}$ can always be identified with Alfvén waves. We expand $\xi$ as a linear combination of $\zeta$, so that

$$
\xi=\sum_{i} Z_{i} \zeta_{i}
$$

where $Z_{i}$ are proportionality constants and $i=l, t$ or $a$. For a uniform field, it is found that the Alfvénic motions get decoupled from the rest and can be treated separately. 


\section{Results}

Equation (1) was solved using a Rayleigh Ritz method (see HS for details). The boundary conditions used were $\xi_{z}=d \xi_{x} / d z=0$ at $z=1000 \mathrm{~km}$ and $\xi_{x}=d \xi_{z} / d z=0$ at $z=-90 \mathrm{~km}$. A constant vertical field $B=2000 \mathrm{G}$, corresponding to $\beta=0.84$ at $z=0$ was used. We first consider the solutions for the $l$ and $t$ modes.

\section{$3.1 l$ AND $t$ MODES}

Figure 2 shows the $\omega-k$ (diagnostic) diagram for umbral oscillations, where $k$ is the horizontal wave number. For a fixed value of $k$, a number of solutions exist satisfying the boundary conditions. These correspond to the normal mode frequencies or harmonics, which form a discrete spectrum. The curves depict the variation of $\omega$ with $k$ for fixed order. We also classify the modes by comparing the magnitudes of the $l$ and $t$ components of $\xi$. Thus, in a $l$ mode $\left|\xi_{l}\right| \gg\left|\xi_{t}\right|$ Open and closed circles correspond to $t$ and $l$ modes respectively, whereas the squares denote mixed modes. In the latter, the first letter denotes the components which as larger magnitude. Physically, the $l$ and $t$ modes can be crudely related to the conventional MHD modes. Above the photosphere, the Alfven speed is much greater than the sound speed. In this case, the fast mode is essentially a transverse mode ( $t$ type) and the slow mode is mainly longitudinal ( $l$ type) for parallel propagation to the field. For perpendicular propagation, however, the fast and slow modes are of the $l$ and $t$ types respectively.

\subsection{ALFVÉN MODES}

The frequencies of the Alfvén modes were also calculated. Since these are independent of the horizontal wave number, we present the results in tabular form, for different mode orders only. Table 1 gives the frequencies and corresponding periods for the lowest five modes.

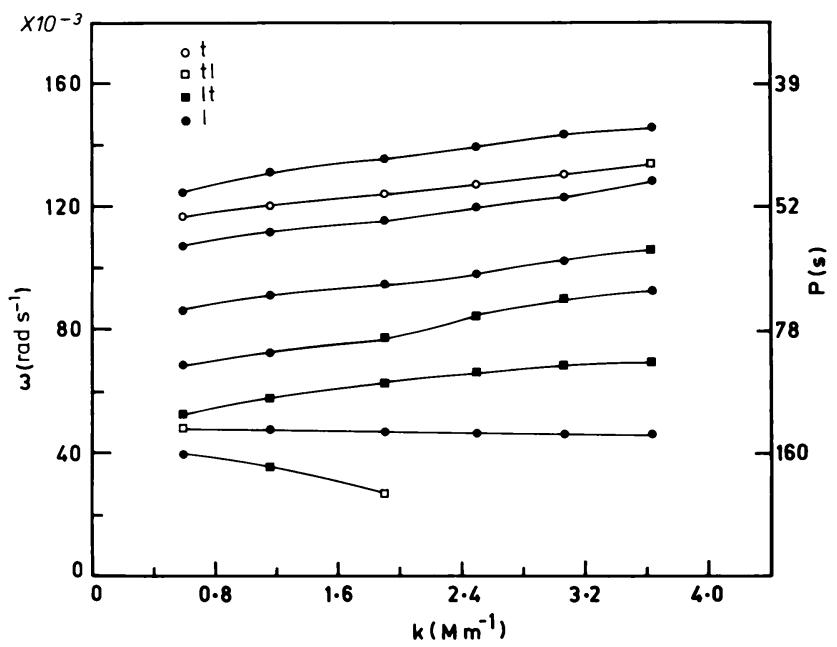

Figure 2. Variation of frequency $\omega$ with $k$ in a sunspot umbra, assuming a uniform vertical magnetic field of 2000G. Open and filled circles denote $t$ and $l$ modes respectively, whereas open and filled squares correspond to mixed modes $t l$ and $l t$ respectively. 
TABLE 1. Alfvén modes in a sunspot umbra

\begin{tabular}{rrr}
\hline$n$ & $\omega\left(s^{-1)}\right.$ & $P(s)$ \\
\hline 1 & 0.025 & 250 \\
2 & 0.098 & 64 \\
3 & 0.160 & 38 \\
4 & 0.230 & 27 \\
5 & 0.310 & 2 \\
\hline
\end{tabular}

\section{Discussion and Conclusions}

We now consider the interpetation of oscillations with periods around $180 \mathrm{~s}$. Assuming an umbral radius of some $3000 \mathrm{~km}$, we find $k=1.3 \mathrm{Mm}^{-1}$ (i.e., for the lowest order mode which has a vanishing radial displacement at the umbral boundary). The corresponding frequencies are $180 \mathrm{~s}$ and $130 \mathrm{~s}$ for the lowest two modes, and are of type $l t$ and $l$ respectively. For parallel propagation above the photosphere, the $130 \mathrm{~s}$ mode resembles a slow wave, whereas the $180 \mathrm{~s}$ modes is a mixed mode, which has a dominant slow component. It should, however, be noted that the nature of the modes changes with $k$ (although the period is comparatively insensitive to $k$ ). We also find that oscillations in the 2-3 min range are unlikely to be of the Alfvenic kind. It is conceivable that the large period (around $300 \mathrm{~s}$ ) oscillations observed for example by Balthasar et al. (1987), might possibly be Alfvén waves in the photosphere. Owing to the rapid increase of the Alfven speed with height, these waves are unlikely to to reach chromospheric levels, since they suffer strong reflection as they propagate upwards.

In conclusion, we have theoretically calculated the spectrum of umbral oscillations. We suggest that the observed modes in the 2-3 min range are of the slow or mixed type. The higher period oscillations may possibly be of the Alfven type.

\section{References}

Abdelatif,T.E.,Lites,B.W., and Thomas,J.H.(1986),Astrophys. J. 311,1015.

Antia,H. and Chitre,S.M. (1979),Solar Phys. 63,67.

Balthasar,H. and Wiehr,E. (1984),Solar Phys. 94,99.

Balthasar,H.,Küveler,G.,and Wiehr,E. (1987),Solar Phys. 112, 39-48.

Beckers,J.M. and Schulz,R.B. (1972),Solar Phys. 27,61.

Bhatnagar,A.,Livingston,W.C., and Harvey,J.W.(1972),Solar Phys. 27,80.

Gurman,J.B. and Leibacher,J.W. (1984), Astrophys. J. 283, 859.

Gurman,J.B. (1987),Solar Phys. 108,61.

Hasan,S.S. and Sobouti,Y. (1987),Mon. Not. R. astr. Soc. 228,427.

Leroy,B. and Schwartz,S.J. (1982),Astron. Astrophys. 112,84.

Lites,B.W. and Thomas,J.H. (1985),Astrophys. J. 294,682.

Lites,B.W. (1986), Astrophys. J. 301,922.

Maltby,P.,Avrett,E.H.,Carlsson,M.,Kjeldseth-Moe,O.,Kurucz,R.L. and Loeser,R. (1986), Astrophys. J. 306,284.

Scheuer,M.A. and Thomas,J.H. (1981),Solar Phys. 71,21.

Schröter,E.H. and Soltau,D. (1976),Astron. Astrophys. 449,463.

Sobouti,Y. (1981), Astron. Astrophys. 100,319.

Soltau,D.,Schröter,E.H., and Wöhl,H. (1976),Astron. Astrophys. 50,367.

Thomas,J.H.,Lites,B.W., and Gurman,J.B. (1987), Astrophys. J. 312,457.

Uchida,Y. and Sakurai,T. (1975),Publ. Astron. Soc. Japan 27,259.

Zhugzhda,Y.D. (1979),Sov. Astron. 23,42.

Zhugzhda,Y.D., Locan, V., and Staude, J. (1983) Solar Phys. 82, 369. 